\title{
Correlation between the numbers of rotation steps in the ATPase and proton-conducting domains of F- and V-ATPases
}

\author{
Hiroyuki Noji ${ }^{1} \cdot$ Hiroshi Ueno ${ }^{1} \cdot$ Ryohei Kobayashi ${ }^{1}$
}

Received: 18 February 2020 / Accepted: 25 February 2020 / Published online: 8 April 2020

(C) The Author(s) 2020

\begin{abstract}
This letter reports the correlation in the number of distinct rotation steps between the $F_{1} / V_{1}$ and $F_{o} / V_{o}$ domains that constitute common rotary F- and V-ATP synthases/ATPases. Recent single-molecule studies on the $\mathrm{F}_{1}$-ATPase revealed differences in the number of discrete steps in rotary catalysis between different organisms -6 steps per turn in bacterial types and mitochondrial $F_{1}$ from yeast, and 9 steps in the mammalian mitochondrial $F_{1}$ domains. The number of rotational steps that $\mathrm{F}_{\mathrm{o}}$ domain makes is thought to correspond to that of proteolipid subunits within the rotating $c$-ring present in $\mathrm{F}_{\mathrm{o}}$. Structural studies on $\mathrm{F}_{\mathrm{o}}$ and in the whole ATP synthase complex have shown a large diversity in the number of proteolipid subunits. Interestingly, 6 steps in $\mathrm{F}_{1}$ are always paired with 10 steps in $F_{o}$, whereas 9 steps in $F_{1}$ are paired with 8 steps in $F_{o}$. The correlation in the number of steps has also been revealed for two types of V-ATPases: one having 6 steps in $V_{1}$ paired with 10 steps in $V_{o}$, and the other one having 3 steps in $\mathrm{V}_{1}$ paired with 12 steps in $\mathrm{V}_{\mathrm{o}}$. Although the abovementioned correlations await further confirmation, the results suggest a clear trend; ATPase motors with more steps have proton-conducting motors with less steps. In addition, ATPases with 6 steps are always paired with proton-conducting domains with 10 steps.
\end{abstract}

\section{ATP synthase}

The ATP synthase, also known as $\mathrm{F}_{\mathrm{o}} \mathrm{F}_{1}$ ATPase or F-ATPase, mediates the energy interconversion between the proton motive force $(\mathrm{pmf})$ across membranes and the free energy of ATP hydrolysis via a rotary catalysis mechanism (Abrahams et al. 1994; Yoshida et al. 2001; Noji et al. 2017). The ATP synthase is composed of two rotary motors, $\mathrm{F}_{1}$ and $\mathrm{F}_{\mathrm{o}}$ (Fig. 1) (Junge et al. 1997). $F_{1}$ is the catalytic core domain responsible for ATP synthesis, showing an active ATPase activity when isolated (Yasuda et al. 2001; Spetzler et al. 2006; Bilyard et al. 2012; McMillan et al. 2016). Upon ATP hydrolysis, $F_{1}$ rotates the inner subunit $(\gamma \varepsilon)$ against the catalytic stator ring $\left(\alpha_{3} \beta_{3}\right)$. $\mathrm{F}_{\mathrm{o}}$ is the membrane-embedded domain and conducts proton translocation across the membrane. Upon proton translocation, $\mathrm{F}_{\mathrm{o}}$ rotates the oligomeric ring formed by the protoncarrying $c$-subunits against the stator complex $\left(a_{2}\right)$. In the whole ATP synthase complex, the rotor parts of $F_{1}$ and $F_{o}$ are bound together, forming the common rotary shaft (Junge

Hiroyuki Noji

hnoji@appchem.t.u-tokyo.ac.jp

1 Applied Chemistry, Graduate School of Engineering, The University of Tokyo, Tokyo 113-8656, Japan et al. 1997; Oster and Wang 2000; Yasuda et al. 2001). The stator parts of $F_{1}$ and $F_{o}$ are connected via the peripheral stalk to transmit the torque without slippage. When pmf is sufficient, $F_{o}$ generates a larger torque than $F_{1}$, reversing the rotation of the rotor shaft in $\mathrm{F}_{1}$ to induce ATP synthesis. In contrast, when $p m f$ is low, $\mathrm{F}_{1}$ reverses the rotation of the rotor ring in $\mathrm{F}_{\mathrm{o}}$, forcing $\mathrm{F}_{\mathrm{o}}$ to actively pump protons and generate $p m f$.

\section{Stepping rotation of $F_{1}$}

The minimum $F_{1}$ complex as a rotary motor is the $\alpha_{3} \beta_{3} \gamma$ subcomplex, which rotates the rod-shaped $\gamma$ subunit against the $\alpha_{3} \beta_{3}$ stator ring in a counterclockwise direction when viewed from the $F_{o}$ side. The catalytic reaction centers for ATP hydrolysis reside at the three pairs of $\alpha-\beta$, with the main catalytic residues harbored in each of the $\beta$ subunits (Weber and Senior 1997). The three $\beta$ subunits conduct the catalytic reaction in a highly sequential manner, resulting in a sequential power-stroking conformational change that rotates the $\gamma$ subunit unidirectionally.

As expected from the pseudo threefold symmetry of $F_{1}$, the unitary rotational step is $120^{\circ}$ rotation, coupled with a single turnover of ATP hydrolysis (Yasuda et al. 1998). The rotation dynamics of the $\gamma$ subunit in $F_{1}$ from thermophilic Bacillus 
Fig. 1 The two rotary motors of ATP synthase, $F_{1}$ and $F_{0}$. The subunit composition of $\mathrm{F}_{1}$ and $\mathrm{F}_{\mathrm{o}}$ in bacterial types is $\alpha_{3} \beta_{3} \gamma \delta \varepsilon$ and $a b_{2} c_{n}$, respectively, where $n$ varies among species. $F_{1}$ rotates the rotary shaft, composed of the $\gamma$ and $\varepsilon$ subunits (red) against the $\alpha_{3} \beta_{3}$ stator ring (blue). $\mathrm{F}_{\mathrm{o}}$ rotates the oligomer ring of the $c$-subunits (red) against the $a b_{2}$ stator complex (blue) during proton translocation across the membrane. In the whole ATP synthase complex, the rotor complexes $\mathrm{F}_{1}$ and $\mathrm{F}_{\mathrm{o}}$ form the common rotary shaft (red) and stator complexes (blue), which are connected via the peripheral stalk formed by the $b_{2}$ and $\delta$ subunits

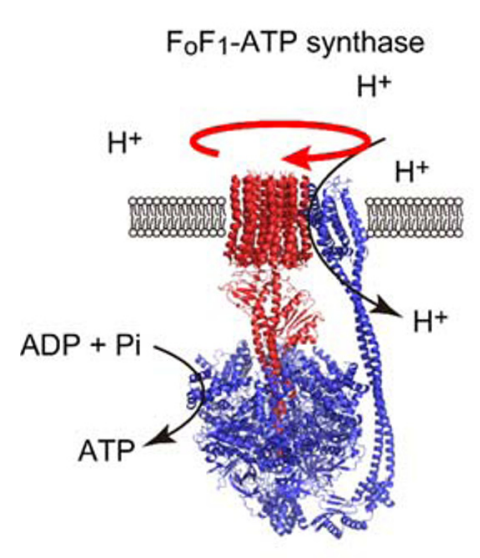

PS3 $\left(\mathrm{TF}_{1}\right)$ has been intensively characterized to establish a standard reaction scheme for bacterial $\mathrm{F}_{1}$ domains. $\mathrm{TF}_{1}$ makes $80^{\circ}$ and $40^{\circ}$ sub-steps in a single $120^{\circ}$ rotation, which means that $\mathrm{TF}_{1}$ makes rotational steps intervened with 6 pauses per turn (Yasuda et al. 2001; Shimabukuro et al. 2003; Nishizaka et al. 2004; Adachi et al. 2007). Other $F_{1}$ domains from bacteria and mitochondrial $\mathrm{F}_{1}$ from yeast $\left(y \mathrm{MF}_{1}\right)$ were reported to make 6 pauses per turn (Steel et al. 2015). Thus, a 6-step rotation is widely conserved across microorganism species.

On the other hand, rotation assays in mammalian $\mathrm{F}_{1}$ domains have found an additional pause in $120^{\circ}$ rotation, which translates into 9 steps per turn (e.g., three step rotations at $65^{\circ}$, $25^{\circ}$, and $\left.30^{\circ}\right)$ in human mitochondrial $\mathrm{F}_{1}\left(h \mathrm{MF}_{1}\right)$ (Suzuki et al. 2014). Similarly, bovine mitochondrial $\mathrm{F}_{1}\left(b \mathrm{MF}_{1}\right)$, the gold standard model for structural analysis of $F_{1}$, was studied in the rotation assay and found to have an additional pause in $120^{\circ}$ rotation (Kobayashi et al. 2020). However, the position in $b \mathrm{MF}_{1}$ is different from $h \mathrm{MF}_{1}$, making three step rotations of $10-20^{\circ}, 60-70^{\circ}$, and $40^{\circ}$. These observations suggest that a 9step rotation is conserved in mammalian mitochondrial $F_{1}$ domains.

We should be able to progressively detect smaller substeps by improving the spatiotemporal imaging resolution and the data analysis methods. In fact, we analyzed the data of rotation trajectories with elaborated mathematical methods and found that $\mathrm{TF}_{1}$ makes an additional small step of $10^{\circ}$ between the $80^{\circ}$ and $40^{\circ}$ sub-steps ( $\mathrm{Li}$ et al. 2015). In this review, we aimed at a coarse-grained classification of the rotation behavior of $F_{1}$. Therefore, we only considered the experimentally distinctive steps: the step size must be over $10^{\circ}$, and/or the intervening pause must be long enough to set the pace of the overall rotation rate under a certain condition, typically in the range of sub- or milliseconds.

\section{Stoichiometry of $\mathrm{H}^{+}$per turn of $\mathrm{F}_{\mathrm{o}}$}

$\mathrm{F}_{\mathrm{o}}$ is a membrane-embedded motor with the minimum subunit composition of $a_{1} b_{2} c_{\mathrm{n}}$. The stoichiometry $(n)$ of the $c$-subunits varies from 8 to 15 among species (Meier et al. 2005; Pogoryelov et al. 2009; Watt et al. 2010; Saroussi et al. 2012; Preiss et al. 2014, 2015; MoralesRios et al. 2015; Guo et al. 2019). The $c$-subunits form an oligomer ring that is rotated against the $a b_{2}$ stator complex upon proton translocation across the membrane. According to the two half-channel model (Vik and Antonio 1994; Junge et al. 1997), which is well supported by the recent cryoEM studies, the $a$-subunit has two halfchannels, one exposed on each side of the membrane (Allegretti et al. 2015). Each proton enters through one of the half-channels and is transferred to one of the $c$ subunits. After one turn of the $c$-ring against the $a b_{2}$ stator, the proton is transferred to the other half-channel of the $a$-subunit facing the opposite side of the membrane. Thus, a proton is translocated by a $c$-subunit, and therefore, the total number of protons translocated per turn is determined by $n$, the number of $c$-subunits in the oligomer $c$-ring.

Currently, there are not enough reports on the stepping rotation of $\mathrm{F}_{\mathrm{o}}$ to discuss the experimental data in a comprehensive manner. Our working assumption is that the number of steps in $\mathrm{F}_{\mathrm{o}}$ is determined by $n$. 


\section{Number of steps in $F_{\mathbf{1}}$ versus $F_{0}$}

We analyzed the data on the following ATP synthases: thermophilic Bacillus PS3 TF, Escherichia coli EF, yeast $y \mathrm{MF}$, and bovine $b \mathrm{MF}$. We chose these ATP synthases because both single-molecule rotation assays on $\mathrm{F}_{1}$ (Watanabe et al. 2010; Bilyard et al. 2012; Steel et al. 2015; Kobayashi et al. 2020) and the structural data on the $c$-ring of $\mathrm{F}_{\mathrm{o}}$ (Stock et al. 1999; Ballhausen et al. 2009; Watt et al. 2010; Guo et al. 2019) are available. Considering the evolutionary distance and the highsequence homology of the $c$-subunits, it is highly likely that the ATP synthase from human mitochondria $(h \mathrm{MF})$ also contains $8 c$-subunits. Therefore, we added the data on $h \mathrm{MF}$. The correlation between the number of steps in $\mathrm{F}_{1}$ and $\mathrm{F}_{\mathrm{o}}$ is shown in Fig. 2. Clearly, a 6-step $F_{1}$ is always paired with a 10-step $F_{o}$, whereas a 9-step $F_{1}$ is paired with an 8-step $F_{o}$.

To gain more data points, we added information gained from the studies on V-ATPases, which are evolutionarily highly related rotary ATPases. V-ATPases are also composed of two distinctive domains, $\mathrm{V}_{1}$ and $\mathrm{V}_{\mathrm{o}}$, corresponding to $F_{1}$ and $F_{o}$, respectively. To date, there are only two wellcharacterized V-ATPases for which the number of rotational steps in $\mathrm{V}_{1}$ and number of proton-carrying units in $\mathrm{V}_{\mathrm{o}}$ is known. One of them is the Enterococcus hirae V-ATPase $(E h \mathrm{~V})$, with a 6-step $\mathrm{V}_{1}$ (Iida et al. 2019) and a $\mathrm{V}_{\mathrm{o}}$ with 10 proton-carrying units (Murata et al. 2005), providing support for the abovementioned correlation. The other one is the V-ATPase from Thermus thermophilus $(T h \mathrm{~V})$, which consists of a 3-step $V_{1}$ (Furuike et al. 2011) and a $V_{o}$ with 12 proton-carrying units (Toei et al. 2007). This data point from $T h \mathrm{~V}$ appears to expand the correlation map to include 3 -step ATPases paired with 12-step proton-conducting domains.

\section{Implications and perspective}

Figure 2 shows an obvious trend: ATPase motors with more steps have proton-conducting motors with less steps. Although the total number of steps varies from 15 to 17 , this trend appears to be relevant in the design principle of rotary ATPases. One possibility is that rotary ATPases are designed to have potential minima around 16 . It is highly likely that some angular pause positions in $\mathrm{F}_{1} / \mathrm{V}_{1}$ overlap with the pause positions in $\mathrm{F}_{\mathrm{o}} / \mathrm{V}_{\mathrm{o}}$. In that case, the above numbers should indicate the maximum numbers of rotary potential minima per turn in the ATPase complex.

In this letter, we only consider the data points of $\mathrm{F} / \mathrm{V}$ ATPases, of which the number of the proteolipid is 8,10 , or 12 , due to the limited information. On the other hand, some ATPase's have different numbers of proteolipids: 9, 11, 13, 14, or 15 (Meier et al. 2005; Pogoryelov et al. 2009; Saroussi et al. 2012; Preiss et al. 2014, 2015). Therefore, it is important to analyze other ATPases to investigate the universality and limitation of the found correlation between the step numbers of $F_{1}$ and $\mathrm{F}_{\mathrm{o}}$. At least, the correlation line in Fig. 2 should be kinked or broken for $\mathrm{F}_{\mathrm{o}} / \mathrm{V}_{\mathrm{o}}$ with proteolipids more than 12 , because the number of rotational steps in $F_{1} / V_{1}$ should not be 2 or less, considering the conservation of the threefold symmetry of $F_{1}$ without exception. A simple expectation is that when the number of proteolipids is 12 or more, $F_{1} / V_{1}$ is a 3-step motor.

In this regard, $\mathrm{F}_{\mathrm{o}} \mathrm{F}_{1}$ from Caldalkalibacillus thermarum TA2.A1 $(C t \mathrm{~F})$ could be along this contention: $C t \mathrm{~F}_{\mathrm{o}}$ has 13 proteolipids in the c-ring (Matthies et al. 2009), and the single-molecule rotation assay of $C t \mathrm{~F}_{1}$ found only 3 distinctive pauses per turn. It should be mentioned that a few rotation trajectories of $C t \mathrm{~F}_{1}$ seem to show a sign of the additional pauses in a turn. A more conclusive analysis is awaited. It
Fig. 2 The number of steps in $\mathrm{F}_{1}$ versus the number of steps in $\mathrm{F}_{\mathrm{o}}$. $T$ F represents data on ATP synthase from thermophilic Bacillus PS3, EF from Escherichia coli, yMF from yeast, $b \mathrm{MF}$ from bovine, $h \mathrm{MF}$ from human, EhV from Enterococcus hirae, and ThV from Thermus thermophilus. Structures of $c_{8^{-}}$ ring of $b \mathrm{MF}$ (orange), $c_{10}$-ring of $T \mathrm{~F}$ (red), $c_{12}$-ring of $T h \mathrm{~V}$ (cyan), and $c_{14}$-ring of Pisum sativum ATP synthase (brown) are shown

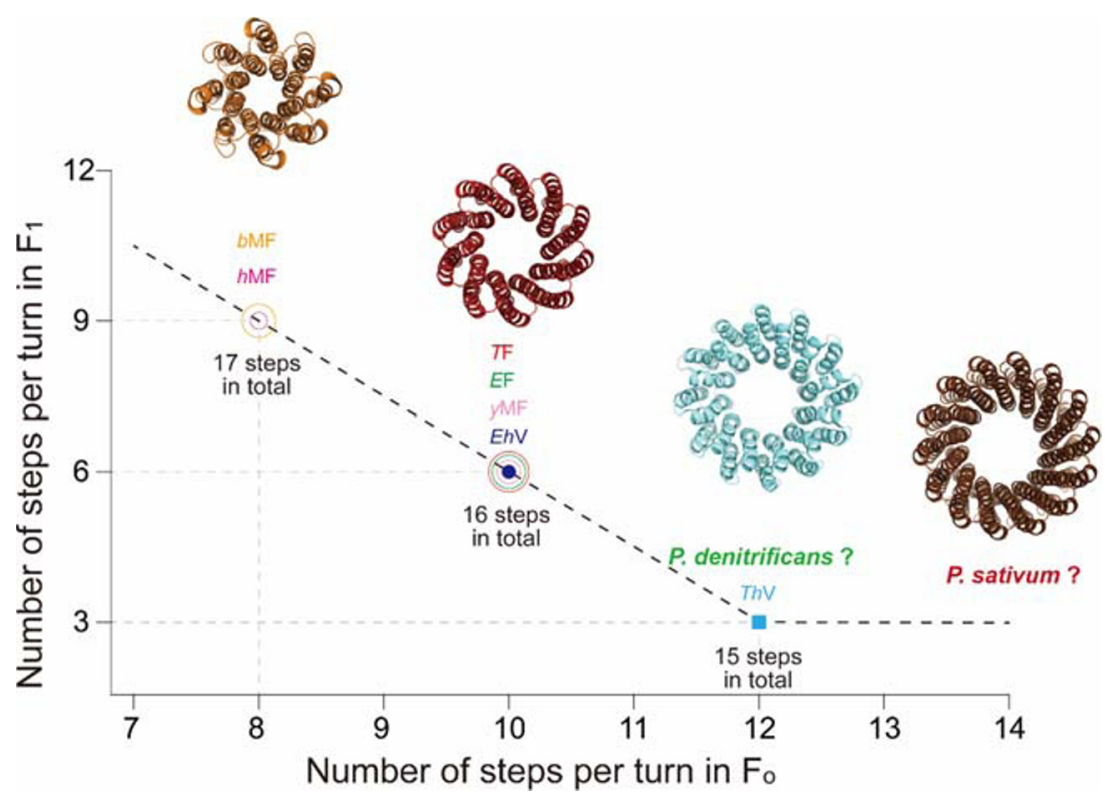


would be also interesting to characterize the rotary catalysis of the F-ATPases from Paracoccus denitrificans (Morales-Rios et al. 2015), Pisum sativum (chloroplast) (Saroussi et al. 2012), and cyanobacteria bacteria species (Pogoryelov et al. 2007 ), in which the $F_{o}$ contains 12,14 , and $13-15 c$-subunits, respectively. It should be noted that the deviance from the found correlation may come from ATPases isolated from cyanobacteria species: the single-molecule rotation assay on a thermophilic cyanobacteria species shows the ADPinhibition pause at a difference position from ATP-binding pause found in active rotation (Konno et al. 2006), suggesting cyanobacterial $F_{1}$ make more than 3 steps per turn.

\section{Summary}

Recent progress in single-molecule rotation analysis and structural analysis on rotary ATPases has revealed a variety of functions and structures among species. This allows for comprehensive analyses. Here, we report a correlation between the number of steps in $F_{1} / V_{1}$ and that in $F_{0} / V_{o}$. There is a clear trend showing that ATPase motors with more steps have proton-conducting motors with less steps. In addition, ATPases with 6 steps are always paired with protonconducting domains with 10 steps. To confirm the universality of these findings, we need more data on the rotation and structure of rotary ATPases. A theoretical approach is also needed to investigate the mechanism behind these rules.

Open Access This article is licensed under a Creative Commons Attribution 4.0 International License, which permits use, sharing, adaptation, distribution and reproduction in any medium or format, as long as you give appropriate credit to the original author(s) and the source, provide a link to the Creative Commons licence, and indicate if changes were made. The images or other third party material in this article are included in the article's Creative Commons licence, unless indicated otherwise in a credit line to the material. If material is not included in the article's Creative Commons licence and your intended use is not permitted by statutory regulation or exceeds the permitted use, you will need to obtain permission directly from the copyright holder. To view a copy of this licence, visit http://creativecommons.org/licenses/by/4.0/.

\section{References}

Abrahams JP, Leslie AGW, Lutter R, Walker JE (1994) Structure at 2.8 A resolution of F1-ATPase from bovine heart mitochondria. Nature 370:621-628. https://doi.org/10.1038/370621a0

Adachi K, Oiwa K, Nishizaka T, Furuike S, Noji H, Itoh H, Yoshida M, Kinosita K (2007) Coupling of rotation and catalysis in F1-ATPase revealed by single-molecule imaging and manipulation. Cell 130 : 309-321. https://doi.org/10.1016/j.cell.2007.05.020

Allegretti M, Klusch N, Mills DJ, Vonck J, Kühlbrandt W, Davies KM (2015) Horizontal membrane-intrinsic $\alpha$-helices in the stator asubunit of an F-type ATP synthase. Nature 521:237-240. https:// doi.org/10.1038/nature14185
Ballhausen B, Altendorf K, Gabriele DH (2009) Constant c10 ring stoichiometry in the Escherichia coli ATP synthase analyzed by crosslinking. J Bacteriol 191:2400-2404. https://doi.org/10.1128/JB. 01390-08

Bilyard T, Nakanishi-Matsui M, Steel BC, Pilizota T, Nord AL, Hosokawa H, Futai M, Berry RM (2012) High-resolution singlemolecule characterization of the enzymatic states in Escherichia coli F1-ATPase. Philos Trans R Soc B Biol Sci 368:2012002320120023. https://doi.org/10.1098/rstb.2012.0023

Furuike S, Nakano M, Adachi K, Noji H, Kinosita K, Yokoyama K (2011) Resolving stepping rotation in Thermus thermophilus H+ATPase/ synthase with an essentially drag-free probe. Nat Commun 2. https://doi.org/10.1038/ncomms1215

Guo H, Suzuki T, Rubinstein JL (2019) Structure of a bacterial ATP synthase. Elife 8. https://doi.org/10.7554/eLife.43128

Iida T, Minagawa Y, Ueno H, Kawai F, Murata T, Iino R (2019) Singlemolecule analysis reveals rotational substeps and chemo-mechanical coupling scheme of Enterococcus hirae V1-ATPase. J Biol Chem 294:17017-17030. https://doi.org/10.1074/jbc.RA119.008947

Junge W, Lill H, Engelbrecht S (1997) ATP synthase: an electrochemical transducer with rotatory mechanics. Trends Biochem Sci 22:420 423. https://doi.org/10.1016/S0968-0004(97)01129-8

Kobayashi R, Ueno H, Li C-B, Noji H (2020) Rotary catalysis of bovine mitochondrial F 1 -ATPase studied by single-molecule experiments . Proc Natl Acad Sci 201909407. https://doi.org/10.1073/pnas. 1909407117

Konno H, Murakami-Fuse T, Fujii F, Koyama F, Ueoka-Nakanishi H, Pack CG, Kinjo M, Hisabori T (2006) The regulator of the F1 motor: inhibition of rotation of cyanobacterial F1-ATPase by the $\varepsilon$ subunit. EMBO J. https://doi.org/10.1038/sj.emboj.7601348

Li CB, Ueno H, Watanabe R, Noji H, Komatsuzaki T (2015) ATP hydrolysis assists phosphate release and promotes reaction ordering in F1ATPase. Nat Commun 6:1-9. https://doi.org/10.1038/ ncomms 10223

Matthies D, Preiss L, Klyszejko AL, Muller DJ, Cook GM, Vonck J, Meier T (2009) The c13 ring from a thermoalkaliphilic ATP synthase reveals an extended diameter due to a special structural region. J Mol Biol. https://doi.org/10.1016/j.jmb.2009.03.052

McMillan DGG, Watanabe R, Cook GM, Ueno H, Noji H (2016) Biophysical characterization of a thermoalkaliphilic molecular motor with a high stepping torque gives insight into evolutionary ATP synthase adaptation. J Biol Chem 291:23965-23977. https://doi.org/ 10.1074/jbc.m116.743633

Meier T, Polzer P, Diederichs K, Welte W, Dimroth P (2005) Structure of the rotor ring of F-type Na+-ATPase from Ilyobacter tartaricus. Science (80- ) 308:659-662. https://doi.org/10.1126/science. 1111199

Morales-Rios E, Montgomery MG, Leslie AGW, Walker JE (2015) Structure of ATP synthase from Paracoccus denitrificans determined by X-ray crystallography at $4.0 \AA$ resolution. Proc Natl Acad Sci U S A 112:13231-13236. https://doi.org/10.1073/pnas.1517542112

Murata T, Yamato I, Kakinuma Y, Leslie AGW, Walker JE (2005) Structure of the rotor of the V-type Na+-ATPase from Enterococcus hirae. Science (80- ) 308:654-659. https://doi.org/ 10.1126/science. 1110064

Nishizaka T, Oiwa K, Noji H, Kimura S, Muneyuki E, Yoshida M, Kinosita K (2004) Chemomechanical coupling in F1-ATPase revealed by simultaneous observation of nucleotide kinetics and rotation. Nat Struct Mol Biol 11:142-148. https://doi.org/10.1038/ nsmb721

Noji H, Ueno H, McMillan DGG (2017) Catalytic robustness and torque generation of the F1-ATPase. Biophys, Rev

Oster G, Wang H (2000) Reverse engineering a protein: the mechanochemistry of ATP synthase. Biochim Biophys Acta Bioenerg 1458: 482-510. https://doi.org/10.1016/S0005-2728(00)00096-7 
Pogoryelov D, Reichen C, Klyszejko AL, Brunisholz R, Muller DJ, Dimroth P, Meier T (2007) The oligomeric state of c rings from cyanobacterial F-ATP synthases varies from 13 to 15 . J Bacteriol. https://doi.org/10.1128/JB.00581-07

Pogoryelov D, Yildiz Ö, Faraldo-Gómez JD, Meier T (2009) Highresolution structure of the rotor ring of a proton-dependent ATP synthase. Nat Struct Mol Biol 16:1068-1073. https://doi.org/10. $1038 /$ nsmb. 1678

Preiss L, Langer JD, Hicks DB, Liu J, Yildiz Ö, Krulwich TA, Meier T (2014) The c-ring ion binding site of the ATP synthase from Bacillus pseudofirmus 0F4 is adapted to alkaliphilic lifestyle. Mol Microbiol 92:973-984. https://doi.org/10.1111/mmi.12605

Preiss L, Langer JD, Yildiz Ö, Eckhardt-Strelau L, Guillemont JEG, Koul A, Meier T (2015) Structure of the mycobacterial ATP synthase Fo rotor ring in complex with the anti-TB drug bedaquiline. Sci Adv 1: 1-9. https://doi.org/10.1126/sciadv.1500106

Saroussi S, Schushan M, Ben-Tal N, Junge W, Nelson N (2012) Structure and flexibility of the C-ring in the electromotor of rotary FoF1ATPase of pea chloroplasts. PLoS One 7:1-12. https://oi.org/10. 1371/journal.pone. 0043045

Shimabukuro K, Yasuda R, Muneyuki E, Hara KY, Kinosita K, Yoshida $\mathrm{M}$ (2003) Catalysis and rotation of F1 motor: cleavage of ATP at the catalytic site occurs in $1 \mathrm{~ms}$ before 40 substep rotation. Proc Natl Acad Sci 100:14731-14736. https://doi.org/10.1073/pnas. 2434983100

Spetzler D, York J, Daniel D, Fromme R, Lowry D, Frasch W (2006) Microsecond time scale rotation measurements of single F1-ATPase molecules. Biochemistry 45:3117-3124. https://doi.org/10.1021/ bi052363n

Steel BC, Nord AL, Wang Y, Pagadala V, Mueller DM, Berry RM (2015) Comparison between single-molecule and X-ray crystallography data on yeast F 1-ATPase. Sci Rep 5:1-10. https://doi.org/10.1038/ srep08773

Stock D, Leslie AGW, Walker JE (1999) Molecular architecture of the rotary motor in ATP synthase. Science (80- ) 286:1700-1705. https://doi.org/10.1126/science.286.5445.1700
Suzuki T, Tanaka K, Wakabayashi C, Saita E, Yoshida M (2014) Chemomechanical coupling of human mitochondrial F1-ATPase motor. Nat Chem Biol 10:930

Toei M, Gerle C, Nakano M, Tani K, Gyobu N, Tamakoshi M, Sone N, Yoshida M, Fujiyoshi Y, Mitsuoka K, Yokoyama K (2007) Dodecamer rotor ring defines H+/ATP ratio for ATP synthesis of prokaryotic V-ATPase from Thermus thermophilus. Proc Natl Acad Sci U S A 104:20256-20261. https://doi.org/10.1073/pnas. 0706914105

Vik SB, Antonio BJ (1994) A mechanism of proton translocation by F1F0 ATP synthases suggested by double mutants of the a subunit. J Biol Chem 269:30364-30369

Watanabe R, Iino R, Noji H (2010) Phosphate release in F 1 -ATPase catalytic cycle follows ADP release. Nat Chem Biol 6:814-820. https://doi.org/10.1038/nchembio.443

Watt IN, Montgomery MG, Runswick MJ, Leslie AGW, Walker JE (2010) Bioenergetic cost of making an adenosine triphosphate molecule in animal mitochondria. Proc Natl Acad Sci U S A 107: 16823-16827. https://doi.org/10.1073/pnas.1011099107

Weber J, Senior AE (1997) Catalytic mechanism of F1-ATPase. Biochim Biophys Acta Bioenerg 1319:19-58. https://doi.org/10.1016/ S0005-2728(96)00121-1

Yasuda R, Noji H, Ishiwata S, Yoshida M, Kinosita K Jr (1998) F1ATPase is a highly efficient molecular motor that rotates with discrete $120^{\circ}$ steps. Cell 93:1117-1124

Yasuda R, Noji H, Yoshida M, Jr KK, Itoh H (2001) Resolution of distinct rotational substeps by submillisecond kinetic analysis of F1ATPase. Nature 410

Yoshida M, Muneyuki E, Hisabori T (2001) ATP synthase - a marvellous rotary engine of the cell. Nat Rev Mol Cell Biol 2:669-677. https:// doi.org/10.1038/35089509

Publisher's note Springer Nature remains neutral with regard to jurisdictional claims in published maps and institutional affiliations. 